\title{
Formation of Non-Potential Magnetic Field and Flare-CMEs Eruption
}

\author{
Hongqi Zhang \\ National Astronomical Observatories, Chinese Academy of Sciences, Beijing 100012, China \\ email:hzhang@bao.ac.cn
}

\begin{abstract}
We analyze the formation process of delta configuration in some well-known super active regions based on the photospheric vector magnetogram observations. It is found that the magnetic field in the initial developing stage of some delta active regions shows the potential-like configuration in the solar atmosphere, the magnetic shear develops mainly near the magnetic neutral line with the magnetic islands of opposite polarities, and the large-scale photospheric twisted field forms late gradually. Some results are obtained: (1) The analysis of magnetic writhe of whole active regions cannot be limited in the strong field of sunspots, because the contribution of the fraction of decayed magnetic field is non-negligible. (2) The magnetic model of kink magnetic ropes, proposed to be generated in the sub-atmosphere, is not consistent with the evolution of large-scale twisted photospheric transverse magnetic field and the relationship with magnetic shear in some delta active regions completely.

The photospheric current helicity density is a quantity reflected the local twisted magnetic field and relates to the remain of transfered magnetic helicity in the photosphere, even if the mean current helicity density brings the general chiral property in a layer of solar active regions. As the emergence of new magnetic flux in active regions, the changes of photospheric current helicity density with the injection of magnetic helicity into the corona from the sub-atmosphere can be detected. Because the injective rate of magnetic helicity and photospheric current helicity density contain the different means in the solar atmosphere, the injected magnetic helicity probably is not proportional to its remain (current helicity density) in the photosphere. A evidence is that the rotation of sunspots does not synchronize with the twist of photospheric transverse magnetic field in some active regions (such as, delta active regions) completely, as one believes that the rotation of sunspots reflects the magnetic one and connects with the injection of magnetic helicity. They represent different aspects of magnetic chirality. The synthetical analysis of the observational magnetic helicity parameters actually provides a relative complete picture of magnetic helicity and its transfer in the solar atmosphere.
\end{abstract}

Keywords. Sun: coronal mass ejections (CMEs), magnetic fields

The vector magnetograms in active region NOAA 5354- 5395-5441-5470 in 1989 are shown in Fig. 1, which were observed at the Huairou Solar Observing Station. It is found that a large dipole active region formed in February 1989 and the tilt angle of active region was about $11^{\circ}$ on Feb. 10 obtained from Solar Geophysical Data. It was a regular magnetic configuration in the early stage of active region. The proceeding magnetic pole of negative polarity broken gradually, as this region appeared again from the eastern limb of the Sun in March. In March it became a typical delta region (Wang et al. 1991; Zhang 1995; Ishii et al. 1998). The main polarity of this region was positive and the magnetic shear of transverse field formed in the vicinity of magnetic neutral line in the eastern side of the magnetic main pole of positive polarity. It is found that the delta configurations only occurred in some evolution stages of the active region and the large-scale twisted magnetic field above the photosphere related to the highly sheared emerging magnetic flux in the active region. 

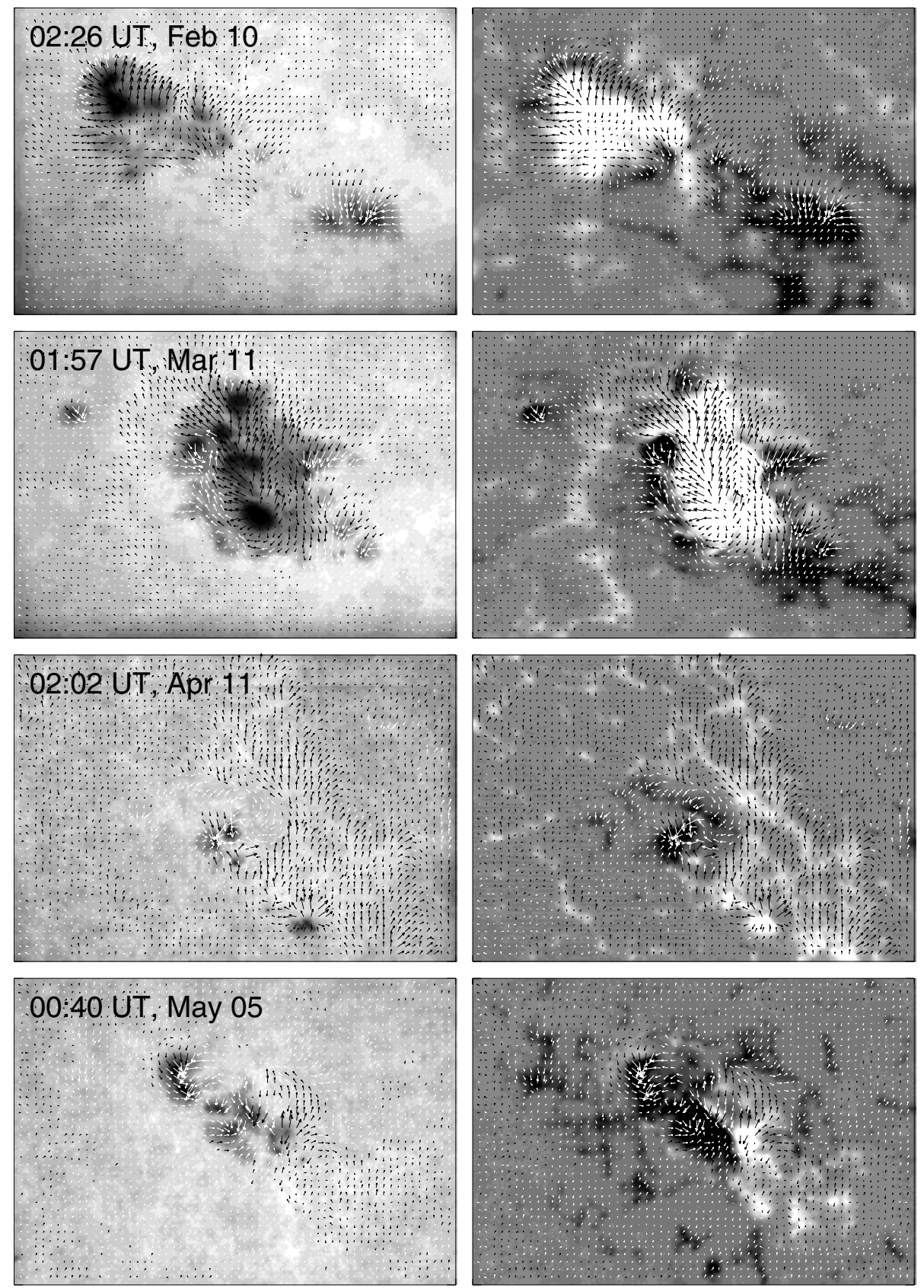

Figure 1. Photospheric images (left) and corresponding vector magnetograms (right) in active region NOAA 5354-5395-5441-5470 in 1989. The white (black) indicates positive (negative) polarity in magnetograms. The arrows indicate the transverse components of field. North is at the top, west is at the right. The size of images is $5.23^{\prime} \times 3.63^{\prime}$.

The electric current increased with the formation of magnetic shear in the active region. By investigating the evolution of magnetic field in the active region, it is found that the formation of local strong current in the eastern side of active region on March 11 was caused by the emergence of magnetic flux with opposite arrangement of magnetic 


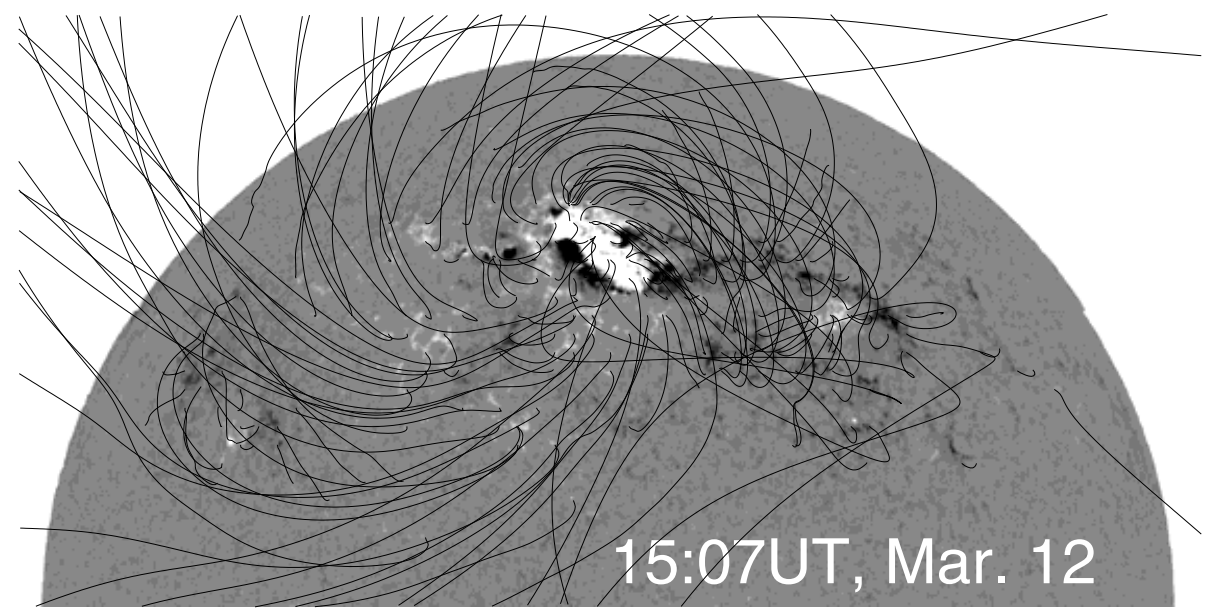

Figure 2. Spatial configuration of magnetic field extrapolated from linear force free field in the north hemisphere based on the MDI full disk magnetogram on 1989 March 12.

polarities. In this active region flares tended to occur near the highly sheared magnetic neutral line, where the transverse field is almost parallel to it, and small scale magnetic features of opposite polarities moved out on either side of the magnetic main pole of positive polarity and curled around it in curved trajectories in the active region (Wang et al. 1991). This means that the magnetic shear and electric current in the solar atmosphere relate to the emergence of magnetic flux of opposite polarity. The flares near the highly sheared magnetic neutral line provide evidence on the reconnection between the emerging magnetic flux and overlying field. In April and May the magnetic main pole of positive polarity broken and the total flux of the active region decreased gradually, while there was also the left handedness of helical magnetic configuration in the active region dominantly. The $\alpha_{\text {best }}$ of force free field is $-1.6 \times 10^{-8} \mathrm{~m}^{-1}$ on Feb. $10,-4.2 \times 10^{-8} \mathrm{~m}^{-1}$ on Mar. 11, $-3.9 \times 10^{-10} m^{-1}$ on Apr. 11 and $-3.0 \times 10^{-8} m^{-1}$ on May 5 , respectively. It is consistent with that the mean sign of current helicity density $h_{c z}$ in the active is negative. Figure 2 shows the magnetic lines of force in the solar atmosphere extrapolated by the linear force free field with $\alpha=-3.8 \times 10^{-8} \mathrm{~m}^{-1}$. It is of a similar order of $\alpha_{\text {best }}$ inferred from photospheric vector magnetogram on Mar. 11. We can find that the most lines of force connect the magnetic main pole of positive polarity and the enhanced network of negative one in the active region. This means that, as the main pole of active region becomes the enhanced network field, the field of active region also keeps the basic bipolar topology obviously in the higher solar atmosphere, even if the field probably is force free, i.e. the current exists in the atmosphere.

The formation of highly sheared magnetic field can be inferred by a series of photospheric vector magnetograms in delta active region NOAA 9077 (Liu and Zhang, 2001; Zhang, 2002). For comparison of the evolution of the highly sheared vector magnetic field, the soft X-ray configuration is shown in Figure 3, the possible formation process of the highly sheared magnetic field above the photosphere in the active region can be inferred. As one believes that the soft X-ray loops at 01:53:04UT on July 11 showed the potential-like field and connected the photospheric footpoints of opposite polarities (even if the prospective effects is significant), while the post soft X-ray loop of "Bastille Day" powerful flare at 17:09:21UT on July 14 provides the relaxed process of magnetic lines of force above the photosphere. The inverse "sigmoid" configuration formed with the decrease of the magnetic shear of photospheric transverse field near the magnetic neutral 


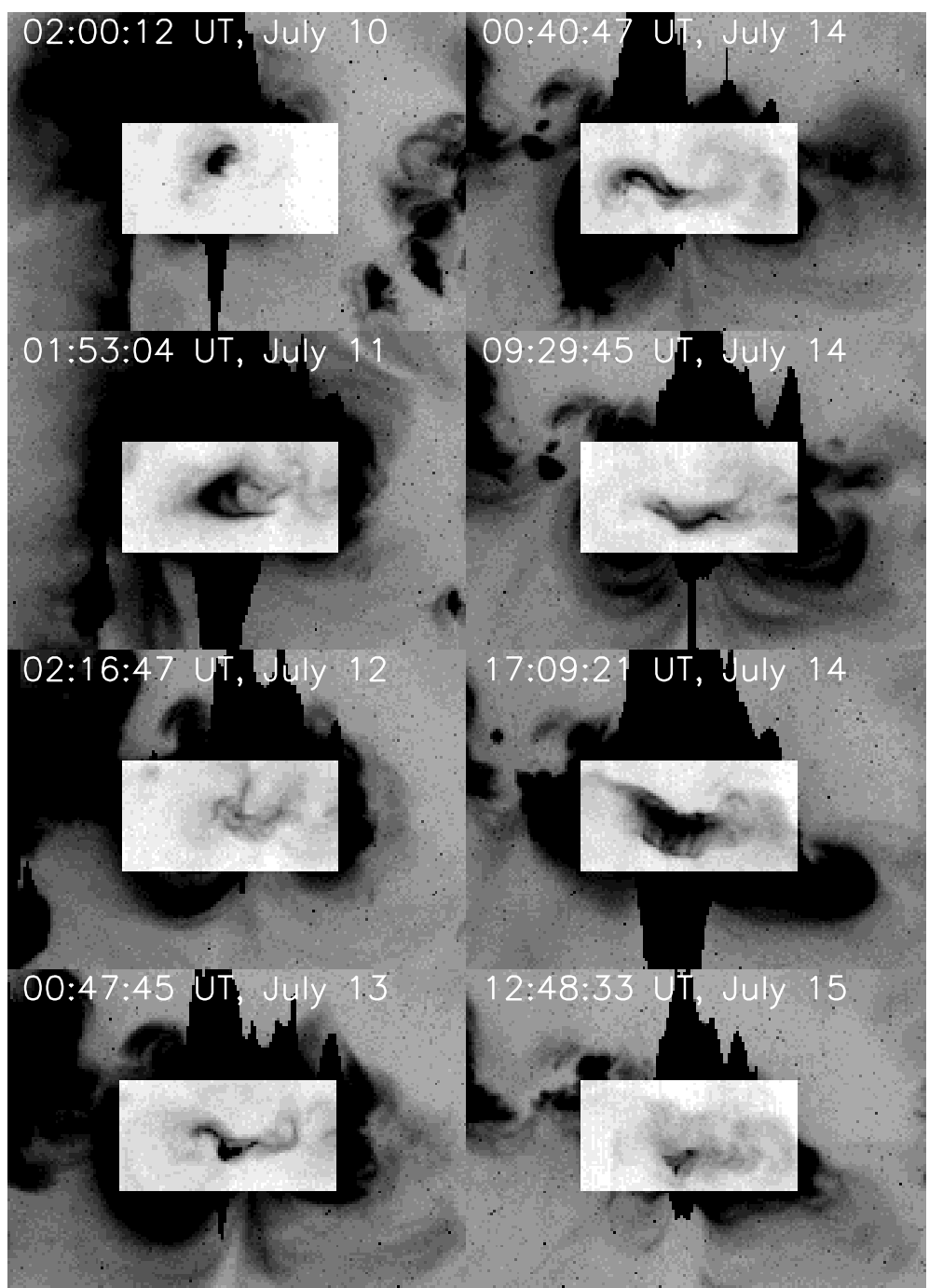

Figure 3. A series of soft X-ray images of active regions NOAA 9077 in 2000 July.

line in the active region on 12 - 14 gradually before the flare. The significant developed inverse "sigmoid" configuration can be found at 00:40:47UT on July 14. It provides an evidence of intense non-potentiality and helicity of magnetic field above the photosphere (Rust and Kumar, 1996; Pevtsov et al., 1997). The mean value of force free field parameter $\alpha$ is about $2.0 \times 10^{-8} m^{-1}$ in the corona, as the estimation of Pevtsov et al. (1997) on the soft X-ray loops has been used. We can conclude that the evolution of photospheric vector magnetic field and corresponding relationship with TRACE $171 \AA$ and soft X-ray features in active region NOAA 9077 provide the formation of that the highly sheared magnetic field transfers from the lower solar atmosphere into the corona in the active region before the "Bastille Day" powerful flare-CME (Yuchyshyn et al., 2001).

The proper motion of sunspot features provides some information on the evolution of magnetic field as the magnetic frozen-in state in the photosphere. One normally can inferred the evolution of magnetic field by the sunspot features. The comparison between the accumulation of magnetic helicity above the photosphere inferred from the 

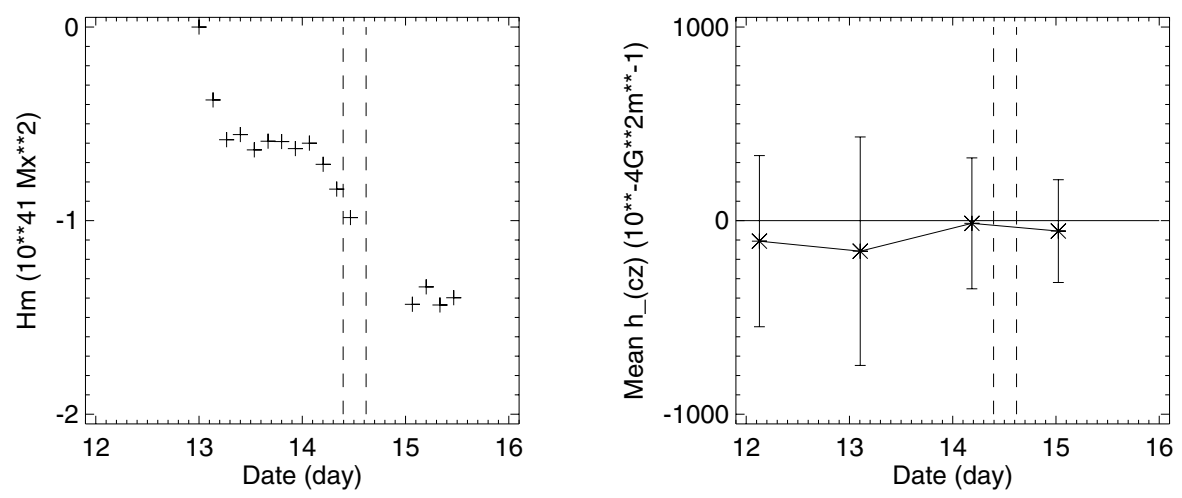

Figure 4. The time profile of the accumulated change of magnetic helicity from the estimated $d H / d t$ (left) and the mean current helicity density $h_{c z}$ (right) in active region NOAA 9077 . The vertical dashed lines mark the begin and end times of "Bastille Day" flare on 2000 July 14.

MDI longitudinal magnetograms by the local correlation tracking techniques and mean photospheric current helicity density $h_{c z}$ in the whole of active region NOAA 9077 is shown in Figure 4. It is found the significant injection of magnetic helicity and the decrease of mean photospheric current helicity density before the "Bastille Day" flare in the active region. It was demonstrated by Zhang (2002) that the high intense current helicity density in the photosphere formed in the vicinity of magnetic neutral line in the active region, on July 12 and 13, and the "Bastille Day" flare-CME on 2000 July 14 occurred after the decay of current helicity density in the photosphere. It is consistent with the injection process of magnetic helicity discussed above, as a whole of the active region. It reflects that the decrease of positive photospheric current helicity density relates the injection of the magnetic helicity from the sub-atmosphere, as comparing the distribution of current helicity density on July 13 and 14 in active region NOAA 9077.

We briefly summarize the main results on the magnetic helicity in the active regions in the following: (1) The magnetic and current helicity commonly provide some similar information on the handedness of twisted magnetic field. It is found that the current helicity evolves with the magnetic helicity in the emerging flux process in active regions. (2) The injection rate of magnetic helicity from the sub-atmosphere and current helicity density in the photosphere contain some different information on the helical properties of magnetic field. The former reflects the transfer process of magnetic helicity, while later does the message of the helical degree of magnetic field and relates to the remain of injected magnetic helicity in the photosphere only. The non-synchronism between them can be found in their evolution process in active regions.

\section{References}

Ishii T., Kurokawa H., Takeuchi T., 1998, ApJ, 499, 989

Liu Y., Zhang H., 2001, A\&\&A, 372, 1019

Pevtsov A., Canfield C., Zirin H., 1997, ApJ, 481, 973

Rust D. and Kumar A., 1996, ApJ, 464, L199

Wang H., Tang F., Zirin H., Ai G., 1991, ApJ, 343, 489

Yurchyshyn V. B., Wang H., Goode P.R. and Deng Y., 2001, ApJ, 563, 381

Zhang H., 1995, A\&AS, 111, 27

Zhang H., 2002, MNRAS, 332, 500 


\section{Discussion}

SCHMiEDER: Does the flux tube emerged twisted or does it become twisted in the upper atmosphere?

ZHANG: Yes, the magnetic flux probably twists in the upper atmosphere, but it is also hard to get obvious evidence how the twist of the magnetic field in the sub-atmosphere from observations of vector magnetic fields.

KusANO: Is the measured magnetic helicity sufficient to activate the kink instability?

ZHANG: The measured magnetic helicity above the solar photosphere in some active regions probably can be used to demonstrate the model of magnetic kink instability. While there is still the problem of finding the evidence that the magnetic ropes formed kinked in the sub-atmosphere before they emerge. 